\title{
INNATE AND ADAPTIVE IMMUNE RESPONSES OF CATFISH ANTIGEN-PRESENTING CELLS TO LIVE ATTENUATED EDWARDSIELLA ICTALURI VACCINES
}

\author{
Adef Kordon $^{1 *}$, Hossam Abdelhamed ${ }^{1 *}$, Wes A Baumgartner ${ }^{2}$, Attila Karsi' ${ }^{1}$, and Lesya M Pinchuk ${ }^{1 \S}$ \\ ${ }^{1}$ Department of Basic Science, Collage of Veterinary Medicine, Mississippi State University, Starkville, \\ MS 39762, United States of America \\ ${ }^{2}$ Department of Pathobiology and Population Medicine, Collage of Veterinary Medicine, Mississippi \\ State University, Starkville, MS 39762, United States of America
}

\begin{abstract}
Efficacious live attenuated Edwardsiella ictaluri vaccines are expected to deliver the antigen to antigenpresenting cells (APCs) that can elicit potent protective innate and adaptive immune responses resulting in the killing of the infected targets or inducing the killing mechanisms in the infected cells. T cell-inducing vaccines must aim to deliver the antigen to APCs so that it can be presented on MHC molecules to the specific T cells. Dendritic cells (DCs) are the most powerful APCs that bridge, control and regulate innate and adaptive immune responses in vertebrates. Recently, we have identified the presence of the Langerhanlike cells containing Birbeck granules in the hemopoietic tissues of channel catfish by the following approaches: flow cytometry, immunohistochemistry, and transmission electron microscopy. Langerin/CD207-positive cells have been detected in catfish spleen and anterior kidney but not in peripheral blood and skin by using mAbs to human Langerin/CD207. These results lay a foundation for our present study that is to determine the vaccine- induced productive innate and adaptive immune responses through catfish APCs, DCs, macrophages, and B cells. Currently, we are evaluating the possible role of Langerin/CD207-positive cells in the protective immunity to $E$. ictaluri vaccines by immunohistochemistry. The function of professional phagocytes, macrophages, to produce nitric oxide in response to live attenuated vaccines are being evaluated by assessing the nitric oxide synthase gene expression levels by real-time PCR. In addition, to characterize the effects of live attenuated vaccines, we are evaluating $\mathrm{T}$ cell-mediated immunity that controls function of APCs and by assessing the gene expression levels of IFN $\gamma$ in peripheral blood, anterior kidney, and spleen of catfish. Finally, the effects of B cell-mediated immunity, B cell-specific gene expression, and immunoglobulin $M$ titers are being determined to live attenuated E. ictaluri vaccines. We suggest that current research will provide the optimal vaccination regime and important basic knowledge on the mechanisms of innate and adaptive immune responses against the most effective vaccines.
\end{abstract}

\section{KEYWORDS}

Edwardsiella ictaluri, Live Attenuated Vaccine, Antigen-Presenting Cells, Dendritic Cells, Catfish

"These authors have contributed equally to this work.

${ }^{\S}$ Corresponding author. Tel.: (662) 325-1436; Fax.: (662) 325-1031.

E-mail address: pinchuk@cvm.msstate.edu 